\title{
INFLUENCIA DO pH E DOS TEORES DE FOSFATO SOLÚVEL E MATÉRIA ORGÂNICA SOBRE A FIXAÇÃo DE ZINCO PELO SOLO*
}

\author{
RONALDO I. SILVEIRA ** \\ F.A.F. DE MELLO ** \\ M.O.C. DO BRASIL SOBRINHO** \\ S. ARZOI $\angle A * *$ \\ J.R. SARKUGE***
}

\section{RESUMO}

No presente trabalho os autores apresentam um estudo dos efeitos do $\mathrm{pH}$ e dos teores de fosfato solúvel em $\mathrm{H}_{2} \mathrm{SO}_{4} 0,05 \mathrm{~N}$ e de matęria orgânica do solo sobre a capacidade de fixação de $\mathrm{Zn}$ do mesmo.

$\mathrm{Na}$ execução do ensaio utilizaram uma técnica traçadora sendo o ${ }^{65} \mathrm{Zn}$ o elemento traçador.

Foi concluído que as quantidades de $\mathrm{Zn}$ fixadas estão direta e positivamente correlacionadas com $\mathrm{o} \mathrm{pH}$, com o teor de fosfato solúvel e de matéria orgânica dos solos.

\section{INTRODUÇÃO}

Em algumas áreas do Estado de São Paulo têm-se verificado deficiência de zinco disponivel principalmente em culturas de milho, batatinha, citros e café. Nestas duas últimas chega a ser, em certos casos, uma prática rotineira a correção de deficiência do referido nutriente por meio de pulverização foliar.

Deve-se ressaltar que em alguns ensaios realizados neste Estado, em que se esperavam reações favoráveis à aplicação de $\mathrm{Zn}$ ao solo, os resultados obtidos não corresponderam às espectativas. Os motivos reais dessa não correspondência são desconhecidos, não estando, porém, afastada a hipótese da fixação do nutriente no solo.

Várias são as causas que concorrem para a fixação do $\mathrm{Zn}$. Neste estudo os autores se propõem a examinar os efeitos do $\mathrm{pH}$ e dos teores de fosfato solúvel e de maté 1 a orgânica do solo.

\section{REVISÃo DA LITERATURA}

A literatura referente à fixação do $\mathrm{Zn}$ no solo é relativamente ampla, ambora no Brasil o assunto não tenha sido motivo de consideração elevada.

\footnotetext{
* Entregue para publicação em 6/10/75. Os autores são gratos aos Profs. E. Malavolta e A. Cervellini pelo fornecimento do ${ }^{65} \mathrm{Zn}$ e pela permissão do uso das dependências e aparelhos do CENA, respectivamente.

* Departamento de Solos da E.S.A. "Luiz de Queiroz".

** Departamento de Química da E.S.A. "Luiz de Queiroz".
} 
Por terem sido investigados na pesquisa aqui relatada 3 fatores que causam ou que concorrem para o fenômeno, julgou-se conveniente grupar os trabalhos encontrados de acordo com os mesmos.

\section{Influência do pH}

Existe, na literatura especializada, um grande número de trabalhos que tratam da relação entre o $\mathrm{pH}$ do solo e a solubilidade e/ou disponibilidade do $\mathrm{Zn}$, a maioria deles indicando existir uma relação inversa entre essas variáveis (CHANDLER, 1937; GALI. \& BARNETTE, 1940; LOTT, 1939; PEECH, 1939;'ROGERS \& CHIH, 1948; THORNE, 1957; CAMP, 1945). Contudo, alguns autores julgam que, mesmo em condições de acidez elevada, pode ocorrer fixação de $\mathrm{Zn}$ devido à formação de compostos portadores desse elemento insolúveis em ácidos (CAMP, et al., 1941). Parece, contudo, não ser essa a regra geral.

À medida que o pH se eleva formam-se no solo compostos que contêm $\mathrm{Zn}$ e cuja solubilidade depende do $\mathrm{pH}$ (DE MUMBRUM \& JACKSON, 1957; JURINAK \& THOR. NE, 1955; NELSON \& MELSTED, 1945; PEECH, 1941). Nas proximidades de pH 7,0 formam-se zincatos de cálcio insolúveis (THORNE, 1957; MALAVOLTA, 1957, pág. 205). Nos solos que contêm carbonatos de $\mathrm{Ca}$ ou de $\mathrm{Mg}$ ou ainda de $\mathrm{Ca}$ e $\mathrm{Mg}$ pode se dar uma fixação muito forte do $\mathrm{Zn}$ na superfíficie desses compostos (SEATZ \& JURINAK, 1957; TISDALE \& NELSON, 1966, pág. 341).

KHAN (1969), WOLTZ et al. (1953), BROWN \& JURINAK (1964), SEATZ et al. (1959), IGUE \& GALLO (1960), constataram em seus experimentos que a calagem acarretava redução na absorção de $\mathrm{Zn}$, chegando às vezes, a causar o aparecimento de sintomas de deficiência desse micronutriente. E, finalmente, LOTT (1939) julga que a toxidez em aveia devido ao excesso de $\mathrm{Zn}$ no solo pode ser evitada pela adição de $\mathrm{CaCO}_{3}$

\section{Influência do teor de fosfato solúvel}

A literatura que trata da fixação do $\mathrm{Zn}$ no solo devido à presença de grandes quan. tidades de íons fosfatados é grande, porém controvertida. Muitos pesquisadores têm encontrado deficiência de $\mathrm{Zn}$ em plantas cultivadas em terrenos com altos teores de fósforo ou que receberam adubações fosfatadas pesadas (BARNETTE et al., 1936; CHAPMAN et al., 1940; ROGERS \& CHIH, 1948; LABANAUSKAS et al., 1958; BINGHAM \& MARTIN, 1956; WOLTZ et al., 1953; BROWN et al., 1970; BOAWN \& LEGGET, 1964; ELLIS et al., 1964).

Utilizando a técnica das soluções nutritivas, CHAPMAN et al. (1937) obtiveram sintomas de deficiência de $\mathrm{Zn}$ em plantas cítricas através da adição de doses crescentes de $\mathrm{P}$ às soluções.

KHAN (1969) concluiu que os sintomas de deficiência de $\mathrm{Zn}$ observados no milho e no arroz nos tratamentos em que se adicionou $P$ eram devidos a um efeito de diluição pois que tais tratamentos proporcionaram produções elevadas. 
BURLESON et al. (1961) sugerem que a deficiência de $\mathrm{Zn}$ causada por excesso de fosfato pode ser, em certos casos, devida a um antagonismo entre $\mathrm{Zn}$ e $\mathrm{P}$ dentro das raizes. Não se trataria, pois de fixação do referido cátion pelo solo.

PEECH (1941) julga não ser o P responsável pela fixação do $\mathrm{Zn}$ nos solos areıosos da Flórida e JAMISON (1944) diz que, naquelas condições, somente aplicações de doses muito elevadas de fertilizantes fosfatados, além dos limites práticos, podem afetar a solubilidade do $\mathrm{Zn}$.

Muitos autores não constataram nenhum efeito ou encontraram efeitos favoráveis do P sobre o aproveitamento do Zn (BOAWN et al., 1954; NIKITIN \& RAINEY, 1952; PEECH, 1941; SEATZ et al., 1959; SHARPLESS et al., 1969; BINGHAM, 1963).

\section{Influência da matéria orgânica}

BARROWS et al. (1960), afirmam que a matéria orgânica é uma das frações do solo mais ativas em transformar o $\mathrm{Zn}$ a formas dificilmente disponiveis às plantas. $\mathrm{E}$ citou diversos autores em apoio a essa tese.

Por outro lado, BERGER \& PRATT (1963), apoiados na revisão bibliográfica efetuada por THORNE (1957), dizem não haver qualquer evidência conclusiva de que a matéria orgânica esteja envolvida na disponibilidade do $\mathrm{Zn}$, embora deficiências desse micronutriente sejam comuns em áreas que tenham recebidos grandes quantidad is de resíduos orgânicos. É que não se pode separar, dizem, os efeitos desses resíduos dos efeitos do $\mathrm{P}$ e de outros constituintes.

BANDYOPADHYA \& ADHIKARI (1968) encontraram uma correlação estreita entre os teores de matéria orgânica do solo e de $\mathrm{Zn}$ extraídos com solução neutra e normal de acetato de amônio.

O que foi dito acima é suficiente para mostrar que pouco se sabe ainda a respeito da influência da matéria orgânica sobre a fixação do $\mathrm{Zn}$.

\section{MATERIAL E MÉTODOS}

Foram utilizadas 30 amostras de solos, sendo 20 do Estado de São Paulo, 4 do Estado de Goiás e 6 do Estado de Mato Grosso, cujos valores pH, teores de fosfato solúvel e de matéria orgânica se acham na Tabela 1. 
TABELA 1 - Valores $\mathrm{pH}$, teores de fosfato solúvel e de matéria orgânica das terras utilizadas no ensaio.

\begin{tabular}{rrrr}
\hline Solo & $\mathrm{pH}$ & $\begin{array}{c}\text { PO }_{4}^{-3} \\
\text { e.mg/100 g T.F.S.A. }\end{array}$ & $\begin{array}{c}\text { Matéria Orgânica } \\
\text { \% na T.F.S.A. }\end{array}$ \\
\hline \hline 1 & 4,8 & 0,03 & 1,65 \\
2 & 6,0 & 0,05 & 1,55 \\
3 & 5,9 & 0,05 & 0,77 \\
4 & 5,1 & 0,03 & 3,10 \\
5 & 5,2 & 0,04 & 1,55 \\
6 & 5,0 & 0,10 & 1,03 \\
7 & 4,8 & 0,02 & 0,87 \\
8 & 4,7 & 0,02 & 0,61 \\
9 & 4,9 & 0,03 & 0,56 \\
10 & 4,7 & 0,02 & 0,92 \\
11 & 5,5 & 0,34 & 3,10 \\
12 & 6,4 & 0,20 & 4,13 \\
13 & 5,2 & 0,16 & 3,10 \\
14 & 5,3 & 0,20 & 4,64 \\
15 & 6,2 & 0,16 & 4,02 \\
16 & 6,2 & 0,25 & 3,87 \\
17 & 5,5 & 0,16 & 2,48 \\
18 & 5,0 & 0,27 & 2,79 \\
19 & 4,4 & 0,12 & 5,83 \\
20 & 6,5 & 0,21 & 1,08 \\
21 & 5,9 & 0,49 & 1,34 \\
22 & 6,0 & 0,45 & 2,06 \\
23 & 6,0 & 0,36 & 2,94 \\
24 & 5,8 & 0,42 & 1,03 \\
25 & 5,9 & 0,38 & 1,03 \\
26 & 6,3 & 0,50 & 3,97 \\
27 & 5,7 & 0,35 & 1,81 \\
28 & 5,6 & 0,35 & 2,12 \\
29 & 5,6 & 0,37 & 1,03 \\
30 & 4,3 & 0,17 & 2,06 \\
\hline
\end{tabular}

Porções de $10 \mathrm{~g}$ de T.F.S.A. foram transferidas para frascos de Erlenmeyer de $250 \mathrm{ml}$, adicionando-se, a seguir, $4 \mathrm{ml}$ de uma solução contendo, nesse volume, $100 \mu \mathrm{g}$ de $\mathrm{Zn}$ inerte sob a forma de sulfato e $0,70 \mu \mathrm{c} \mathrm{de}{ }^{65} \mathrm{Zn}$ sob a forma de cloreto.

Os frascos foram cobertos com papel de filtro e deixados em repouso durante 20 dıas, sob as condições normais de laboratório. Após esse período, procedeu-se à extração do $\mathrm{Zn}$ utilizando-se $20 \mathrm{ml}$ de uma solução $0,01 \mathrm{M}$ em EDTA e $1 \mathrm{M}$ em $\left(\mathrm{NH}_{4}\right)_{2} \mathrm{CO}_{3}$ ajustada a pH 8,6, de acordo com a técnica empregada por TRIERWEILLER \& LINDSAY (1969).

Alíquotas de $5 \mathrm{ml}$ dos extratos foram transferidas para tubos de ensaio a fim de se proceder à determinação das atividades do ${ }^{65} \mathrm{Zn}$ nelas contido, em contador por cintilação sólida. $\mathrm{Na}$ mesma ocasião determinou-se a atividade do ${ }^{65} \mathrm{Zn}$ contido em $5 \mathrm{ml}$ da solução original e que serviu como padrão. 
A partir das contagens procedidas sobre o padrão e extrator foram calculadas as quantidades de $\mathrm{Zn}$ extraidas. As diferenças entre as quantidades aplicadas e as extraídas foram consideradas como fixadas, de acordo com o conceito apresentado por KARDOS (1955).

Foram feitos estudos de correlação e calculadas as equações de regressão linear entre as quantidades de $\mathrm{Zn}$ fixadas e os valores encontrados de $\mathrm{pH}$, fosfato solúvel e matéria orgânica, de acordo com PIMENTEL GOMES (1970, pág. 295-313).

As determinações de $\mathrm{pH}$, do fosfato solúvel e de matéria orgânica foram efetuadas pelos seguintes métodos:

pH - potenciométrico, com relação terra-água igual a 1-2,5;

fosfato solúvel - fotocolorimétrico, usando como extrator uma solução $0,05 \mathrm{~N}$ em $\mathrm{H}_{2} \mathrm{SO}_{4}$ e ácido ascórbico como redutor;

matéria orgância - via úmida, por oxidação com bicromato de potássio em meio acidificado por ácido sulfúrico.

\section{RESULTADOS E DISCUSSÃO}

As quantidades de $\mathrm{Zn}$ que foram fixadas por $10 \mathrm{~g}$ dos diversos solos empregados estão apresentados na Tabela 2.

\section{Influência do pH}

Conforme foi relatado na revisão de literatura, há um acordo geral entre os autores de que o $\mathrm{pH}$ do solo exerce uma ação muito importante sobre a solubilidade do $\mathrm{Zn}$ e sua assimilabilidade pelas plantas, o que significa que o $\mathrm{pH}$ exerce influência notável sobre a capacidade de fixação de $\mathrm{Zn}$ pelo solo.

Estudando-se a relação entre $\mathrm{pH}$ e quantidades de $\mathrm{Zn}$ fixadas pelas terras (Tabelas 1 e 2) foi encontrado um coeficiente de correlação $r=0,49^{* *}$, significativo a $1 \%$ e a equação de regressão seguinte:

$$
y=-17,09+6,59 x
$$

em que y é a quantidade de $\mathrm{Zn}$ fixada e $\mathrm{x}$ o $\mathrm{pH}$ correspondente. Pode-se observar que o pH afetou a fixação do $\mathrm{Zn}$, esta aumentando à medida que aquele aumentou e vice-versa.

De acordo, ainda, com a equação (1) a fixação deixaria de existir a pH 2,6, incompatível com solos cultivados. 
TABELA 2 - Quantidades de $\mathrm{Zn}$ fixadas pelos diversos solos.

\begin{tabular}{rr}
\hline Solo & $\begin{array}{c}\text { Quantidade de Zn fixada } \\
\mu \mathrm{g} / 10 \mathrm{~g} \text { T.F.S.A. }\end{array}$ \\
\hline \hline 1 & 11,62 \\
2 & 9,47 \\
3 & 12,43 \\
4 & 6,98 \\
5 & 17,43 \\
6 & 16,69 \\
7 & 10,76 \\
8 & 12,84 \\
9 & 14,43 \\
10 & 9,06 \\
11 & 18,15 \\
12 & 30,86 \\
13 & 27,05 \\
14 & 36,62 \\
15 & 20,89 \\
16 & 30,30 \\
17 & 15,95 \\
18 & 20,23 \\
19 & 14,81 \\
20 & 24,09 \\
21 & 22,21 \\
22 & 26,19 \\
23 & 37,29 \\
24 & 11,19 \\
25 & 18,14 \\
27 & 30,80 \\
28 & 18,51 \\
29 & 24,09 \\
30 & 7,72 \\
\hline & 12,43 \\
\hline
\end{tabular}

Estudando o fenômeno de outra forma, BANDYOPADHYA \& ADHIKARI (1968) encontraram nos solos com que trabalharam uma estreita relação entre os teores de $\mathrm{Zn}$ extraídos com solução de acetato de amônio neutra e normal e os pHs respectivos. Tal relação se caracterizava por um coeficiente de correlação $r=0,7911^{* *}$, sendo a equação de regressão linear $y=11,4104-1,4376 \mathrm{x}$. De acordo com essa equação, a um pH aproximadamente igual a 7,9 não haveria remoção de $\mathrm{Zn}$ das terras. Isso está de acordo com THORNE (1957) que, citando vários autores, diz ser significativa a quantidade de zincatos em solos com pH superior a 7,85.

$\mathrm{O}$ efeito do $\mathrm{pH}$ parece depender da natureza do cátion predominante, como sugere o trabalho de JURINAK \& THORNE (1955). Esses autores estudaram a possibilidade de formação de zincatos em solos alcalinos. Para isso, fizeram variar o $\mathrm{pH}$ de suspensões de argila contendo $\mathrm{Zn}$, por meio de adições de hidróxidos de $\mathrm{Na}$, de $\mathrm{K}$ e de $\mathrm{Ca}$. 
Verificaram que, nos dois primeiros casos, a solubilidade do $\mathrm{Zn}$ era mínima quando o pH das suspensões estava entre 5,5 e 6,7, aumentando com posterior diminuição da concentração hidrogeniônica, o que sugere a formação de zincatos alcalinos insolúveis entre aquela faixa de $\mathrm{pH}$.

Entretanto, quando se empregou o hidróxido de $\mathrm{Ca}$ para elevar o $\mathrm{pH}$ das suspensões, a solubilidade do $\mathrm{Zn}$ atingiu um mínimo a $\mathrm{pH} 7,6$, não aumentando com posterior elevação deste. Daí os autores postularem a formação de zincato de $\mathrm{Ca}$ insolúvel.

Alguns autores (CAMP et al., 1941; THOMPSON, 1957, pág. 342) expressaram a opinião de que também em condições ácidos a solubilidade do $\mathrm{Zn}$ pode decrescer. CAMP et al. (1941) julgaram que a deficiência desse nutriente observada na Flórida em solos com pH entre 4,5 a 5,25 se devia à formação de compostos insolúveis em ácidos. Porém não fizeram menção à natureza de tais compostos.

Como no experimento ora apresentado o pH das terras variou de 4,3 a 6,5, deve-se supor que existem nos solos vários processos dependentes da reação dos mesmos e que são responsáveis pela retenção do $\mathrm{Zn}$ operando simultaneamente ou não. Essa é, também, a opinião de BARROWS et al. (1960).

WEAR (1956) tratou porções de terra com diferentes doses de $\mathrm{CaCO}_{3}, \mathrm{Na}_{2} \mathrm{CO}_{3} \mathrm{e}$ $\mathrm{CaSO}_{4}$, plantando sorgo em seguida. Os dois primeiros sais elevaram o $\mathrm{pH}$ da terra reduzindo a absorção de $\mathrm{Zn}$ pelas plantas. $\mathrm{O}$ último, contudo, causou um abaixamento do $\mathrm{pH}$ e maior absorção de $\mathrm{Zn}$, embora o teor de $\mathrm{Ca}$ da plantas também aumentem. $\mathrm{O}$ autor concluiu que o $\mathrm{pH}$ era importante na absorção do $\mathrm{Zn}$ e não o $\mathrm{Ca}$.

É interessante citar também o experimento de VIETS et al. (1957) que comparando as eficiências de fontes de $\mathrm{N}$ (sulfato de amônio, nitrato de amônio e nitrato de sódio) sobre a absorção por plantas do $\mathrm{Zn}$ natural do solo e do $\mathrm{Zn}$ aplicado como sulfato, concluíram que as diferenças observadas poderiam ser explicadas pelas alterações causadas no $\mathrm{pH}$ da terra pelos adubos. Concluíram, também, que a absorção do $\mathrm{Zn}$ nativo do solo era maior quando se empregava sulfato de amônio que quando se empregava nitrato de sódio. É sabido que o primeiro tende acidificar o solo (CATANI \& GALLO, 1954; NEVES et al., 1960; CHICA \& LOTERO, 1969).

Observa-se, pois, que o resultado obtido no trabalho ora apresentado sobre o efeito do $\mathrm{pH}$ na fixação do $\mathrm{Zn}$ está de acordo com o que informa a literatura.

\section{Influéncia do teor de fosfato no solo}

$\mathrm{O}$ confronto dos dados das Tabelas 1 e 2 permitiu chegar-se à verificação de que existe uma relação estreita entre os teores de $\mathrm{PO}_{4}^{-3}$ das terras utilizadas neste ensaio e as quantidades de $\mathrm{Zn}$ fixadas pelos mesmos. Foi encontrado um coeficiente de correlação $r=0,58^{* *}$, significativo a $1 \%$, sendo:

$$
y=12,62+31,05 x
$$

a equação de regressão que liga essas variáveis. Nela, y representa as quantidades de $\mathrm{Zn}$, em $\mu \mathrm{g}$, fixadas por $10 \mathrm{~g}$ de T.F.S.A. e $\mathrm{x}$ o número de e.mg de $\mathrm{PO}_{4}^{-3}$ por $100 \mathrm{~g}$ de T.F.S.A. 
Aparentemente, a explicação mais plausível para o fenômeno acima é uma precipitação do $\mathrm{Zn}$ adicionado pelo íon fosfato. É o que se pode supor com base nos resultados obtidos por JAMISON (1944), citado por THORNE (1957). Aquele autor verificou que a solubilidade do $\mathrm{Zn}$ em solução de fosfato decrescia à medida que aumentava a concentração deste. Possivelmente, esse é o motivo porque CHAPMAN et al. (1937) conseguiram obter e intensificar os sintomas de deficiência de $\mathrm{Zn}$ em plantas cítricas cultivadas em soluções nutritivas, unicamente pela adição às soluções de doses crescentes de fosfato.

Entretanto, como se pode apreciar anteriormente, o assunto é complexo e controvertido; SHARPLESS et al. (1969) não constataram nenhuma significância na relação entre o conteúdo de $\mathrm{P}$ dos solos e as capacidades de fixação de $\mathrm{Zn}$ dos mesmos. Esses motivos levaram THORNE (1957) a declarar que não se pode explicar as deficiências de $\mathrm{Zn}$ observadas em plantas somente através de precipitação direta do mesmo pelos ions fosfato.

\section{Influência da matéria orgánica}

Os cálculos efetuados envolvendo os teores de matéria orgânica e as capacidades de fixação de $\mathrm{Zn}$ das terras utilizadas neste ensaio (Tabelas 1 e 2) revelaram existir uma relação muito intima entre essas duas séries de variáveis, revelada por um coeficiente de correlação $r=0,54^{\star \star}$, significativo a $1 \%$. A equação de regressão linear encontrada foi:

$$
y=11,65+3,29 x
$$

sendo y a quantidade, em $\mu \mathrm{g}$, de $\mathrm{Zn}$ fixada por $10 \mathrm{~g}$ de T.F.S.A. e $\mathrm{x}$ o seu teor em matéria orgânica. A equação de regressão linear informa, que as quantidades de $\mathrm{Zn}$ fixadas pelas terras aumentaram de acordo com o conteúdo orgânico das mesmas.

Esse resultado está de acordo com o que foi observado por outros autores.

$O$ único trabalho encontrado que está em desacordo com o consenso geral é o de BANDYOPADHYA \& ADHIKARI (1968) que acharam uma correlação positiva e significativa ao nível de $1 \%$ entre os teores de $\mathrm{Zn}$ extraível com solução de acetato de amônio neutra e normal e os teores de matéria orgânica dos solos estudados.

As explicações geralmente oferecidas para justificar o aumento da capacidade de fixar $\mathrm{Zn}$ das terras em função do teor de matéria orgânica, têm-se relacionado com microrganismos e com a formação de quelatos e de outras substâncias organo-metálicas complexas, cujo metal é o $\mathrm{Zn}$. 


\section{CONCLUSÕES}

A intenção dos autores, ao executarem este trabalho, foi a de verificar as influências do $\mathrm{pH}$ e do teor de fosfato solúvel e de matéria orgânica do solo sobre a capacidade de fixação de zinco do mesmo.

Para isso, porções de $10 \mathrm{~g}$ de 30 amostras de diferentes solos foram transferidas para frascos de Erlenmeyer de $250 \mathrm{ml}$, adicionando-se, a seguir, $4 \mathrm{ml}$ de uma solução contendo $100 \mu \mathrm{g}$ de $\mathrm{Zn}$ inerte e $0,7 \mu \mathrm{c} \mathrm{de}{ }^{65} \mathrm{Zn}$.

Após 20 dias de incubação fez-se a extração do $\mathrm{Zn}$ com $20 \mathrm{ml}$ de uma solução $1 \mathrm{Mi}$ em $\left(\mathrm{NH}_{4}\right)_{2} \mathrm{CO}_{3}$ e $0,01 \mathrm{M}$ em EDTA.

Alíquotas de $5 \mathrm{ml}$ dos extratos foram passadas para tubos de ensaio para a determinação da atividade do ${ }^{65} \mathrm{Zn}$ contido nas mesmas em contador de cintilação. A partir dos dados obtidos e da contagem procedida sobre uma solução padrão procedeu-se ao cálculo da quantidade de $\mathrm{Zn}$ fixada pelas diferentes terras.

Os resultados obtidos permitem concluir que as quantidades de $\mathrm{Zn}$ fixadas estão direta e positivamente correlacionadas corn o $\mathrm{pH}$, com o teor de fosfato solúvel e de matéria orgânica dos solos.

\section{SUMMARY}

\section{EFFECTS OF pH, SOLUBLE PHOSPHATE AND ORGANIC MATTER ON ZINC FIXATION BY SOIL}

The purpose of this experiment was to verify the effects of some properties of the soil as $\mathrm{pH}$, and the content of soluble phosphate and organic matter on the soil capacity to fix zinc.

Ten grams each of 30 samples of different soils were placed in a $250 \mathrm{ml}$ Erlenmeyer flask and $4 \mathrm{ml}$ of solution containing $100 \mu \mathrm{g}$ of inert zinc and $0.7 \mu \mathrm{c}$ of radioactive ${ }^{65} \mathrm{Zn}$ were added. After 20 days of incubation, $\mathrm{Zn}$ was extracted with $20 \mathrm{ml}$ of $0.01 \mathrm{M}$ EDTA solution made $1 \mathrm{M}$ respect to $\left(\mathrm{NH}_{4}\right)_{2} \mathrm{CO}_{3}$. An aliquot of $5 \mathrm{ml}$ of the extract was transferred to a test tub and radioactivity of ${ }^{65} \mathrm{Zn}$ in it was measured with a scintillation counter. Quantity of zinc fixed by the different soils was calculated from the data obtained and county made on the standard solution.

It was concluded that the amount of zinc fixed by the soil was positively correlated with $\mathrm{pH}$, soluble phosphate and organic matter content of the soils. 


\section{LITERATURA CITADA}

BANDYOPADHYA, A.K. \& ADIKARI, M., 1968. Trace element relationships in rice soils: I - Alluvial soils of west Bengal. Soil Sci., 105:224-247.

BARNETTE, R.M., CAMP, J.P., WAGNER, J.D. \& GALL, O.E., 1936. The use of zinc sulfate under corn and other fild crops. Florida Agric. Exp. Sta. Bull. 292.

BARROWS, H.L., NEFF, M.F. \& GAMMON JR., N., 1960. Effect of soil type on mobility of zinc in the soil and on its availability from zinc sulfate to tung. Soil Sci. Soc. Am. Proc., 24:367-372.

BERGER, K.C. \& PRATT, P.F., 1963. Advances in secondary and micronutrient fertilization. Em: Fertilizer Technology and Usage. Soil Sci. Soc. of America, Madison II, Wisconsin, pág. 287-340.

BINGHAM, F.T. \& MARTIN, J.P., 1957. Effects of soil phosphorus on growth and minor element nutrition of citrus. Soil Sci. Soc. Am. Proc., 20:382-385.

BINGHAM, F.T., 1963. Relation between phosphorus and micronutrients in plants. Soil Sci. Soc. Am. Proc., 27:389-391.

BOAWN, L.C. \& LEGGET, G.E., 1964. Phosphorus and zinc concentrations in Russet Binbank potato tissues in relation to development of zinc symptoms. Soil Sci. Soc. Proc., 28:229-232.

BOAWN, L.C., VIETS, F.G. \& CRAWFORD, C.L., 1954. Effect of phosphate fertilizers on zinc nutrition of field beans. Soil Sc., 78:1-17.

BROWN, A.L. \& JURINAK, J.J., 1964. Effect of liming on the availability of zinc and copper. Soil Sci., 98:170-173.

BROWN, A.L., KRANTZ, B.A. \& EDDINGS, J.L., 1970. Zinc - phosphorus interactions as measured by plant response and soil analysis. Soil Sci., 110:415-420.

BURLESON, C.A., DACUS, A. \& GERARD, C.J., 1961. The effect of phosphorus fertilization on the zinc nutrition of several irrigated crops. Soil Sci. Soc. Am. Proc., 25:365-368.

CAMP, A.F., CHAPMAN, H.D., BAHRT, G.M. \& PARKER, E.R., 1941. Symptoms of citrus malnutrition. Em: Hunger signs in crops, cap. 9. The American Society of Agronomy and the National Fertilizer Association, Washington, D.C.

CAMP, A.F., 1945. Zinc as a nutrient in plant growth. Soil Sci., 60:157-164.

CATANI, R.A. \& GALLO, J.R., 1954. Efeitos determinados no solo pelo uso contínuo de fertilizantes. Bragantia, 13:75-83.

CHANDLER, W.H., 1937. Zinc as a nutrient for plants. Bot. Gaz., 98:625-646.

CHAPMAN, H.D., LIEBIG JR. G.F., \& VANSELOU, A.P., 1940. Some nutritional relationships on revealed by a study of mineral deficiency and excess symptoms on citrus. Soil Sci. Soc. Am. Proc., 4:196-200.

CHICA, G., JAVIER \& JAIME LOTERO, C., 1969. Influencia de fuentes y dosis de nitrogeno en el pH de un suelo aluvial. Rev. do Inst. Colombiano Agropecuário, 4:31-49.

DE MUMBRUM, L.E. \& JACKSON, M.L., 1957. Formation of basic cations of copner.zinc, iron and aluminum. Soil Sci. Soc. Am. Proc., 21:662.

ELLIS, R. JR., DAVIS, J.E. \& THURLOV, D.L., 1964. Zinc availability in calcareous Michigan soils as influenced by phosphorus and temperature. Soil Sc. Soc. Am. Proc., 28:83-86.

GALL, O.E. \& BARNETTE, R.M., 1940. Toxic limits of replaceable zinc to corn and cowpeas grown on three Florida soils. J. Am. Soc. Agron., 32:23-32.

IGUE, K. \& GALLO, J.R., 1960. Zinc deficiency of corn in São Paulo. IBEC Research Institute, Bull. 20.

JAMISON, J.S., 1944. The effect of phosphate upon the fixation of zinc and copper in several 
Florida soils. Proc. Fla. State Hort. Sci., 26-31.

JURINAK, J.J. \& THORNE, D.W., 1955. Zinc solubility under alkaline conditions in a zinc-bentonite system. Soil Sci. Soc. Am. Proc., 19:446-448.

KARDOS, L.T., 1955. Em: Chemistry of the soil. Editado por F.E. Bear Reinnold Publishing Corporation, New York.

KHAN, D.H., 1969. Response of sweet corn and rice to phosphorus, zinc and calcium carbonate on acid glenview soil of California. Soil Sci., 108:424-428.

LABANAUSKAS, C.K., EMBLETON, T.W. \& JONES, W.W., 1958. Influence of phosphate fertilizers on micronutrients in avocado leaves subject to longterm study of finest orchard. Calif. Agric., 12:10.

LOTT, W.L., 1939. The relation of hidrogen ion concentration to the availability of zinc in the soil. Soil Sci. Soc. Am. Proc., $3: 115-121$.

NELSON, J.L. \& MELSTED, S.W., 1955. The chemistry of zinc added to soil and clays. Soil Sci. Soc. Am. Proc., 19:439-443.

NEVES, O.S., VIEGAS, G.P. \& FREIRE, E.S., 1960. Efeito do uso contínuo de certos adubos azotados sobre o pH do solo. Bragantia, 19 (2 parte), nota no 25.

NIKITIN, A.A. \& RAINEY, J.W., 1952. Reactions between trace element salts and N-P-K carriers in fertilizers. Agron. J., 44:541-546.

PEECH, M., 1939. Chemical studies on soil from Florida citrus groves. Florida Agric. Exp. Sta. Tech. Bull. 340.

PEECH, M., 1941. Availability of ions in light sandy soils as affected by soil reaction. Soil Sci., $51: 473-486$.

PIMENTEL GOMES, F., 1970. Em: Curso de Estatística Experimental, 4ạ edição. Escola Superior de Agricultura "Luiz de Queiroz", Piracicaba.

ROGERS, L.H. \& CHIH, WV, 1948. Zinc uptake by oats as influenced by application of lime and phosphate. J. Am. Soc. Agron., 46:563-566.

SEATZ, L.F. \& JURINAK, J.J., 1957. Zinc and soil fertility. Em: Soil, the 1957 Yearbook of Agriculture, pág. 115-121.

SEATZ, L.F., STERGES, A.J. \& KRAMER, J.C., 1959. Crops response to zinc fertilization as influenced by lime and phosphorus applications.

SHARPLESS, R.G., WALLIHAN, E.F. \& PETERSON, F.F., 1969. Retention of zinc by some arid zone soil materials treated with zinc sulfate. Soil Sci. Soc. Am. Proc., 33:901-904.

TISDALE, S.L. \& NELSON, W.L., 1966. Soil Fertility and Fertilizers, 2a ed. The MacMillan Co., New York; Collier MacMillan Ltd. London.

THORNE, W., 1957. Zinc deficiency and its control. Adv. Agron., 9:31-65.

TRIERWELLER, J.F. \& LINDSAY, W.L., 1969. EDTA - ammonium carbonate soil test of zinc. Soil Sc. Soc. Am. Proc., 33:49-53.

WOLTZ, S., TO'TH, S.J. \& BEAR, F.E., 1953. Zinc status of New Jersey soils. Soil Sci., 76:115-122 . 
